\title{
Artemether Targets Raptor-Induced Actin Polymerization and Suppresses Migration of Fibroblast-like Synoviocytes in Rheumatoid Arthritis
}

Jian-Da Ma

Sun Yat-Sen Memorial Hospital

Jun Jing

Sun Yat-Sen Memorial Hospital

Jun-Wei Wang

Sun Yat-Sen Memorial Hospital

Xue-Pei Zhang

Sun Yat-Sen Memorial Hospital

Qian-Hua Li

Sun Yat-Sen Memorial Hospital

Ying-Qian Mo

Sun Yat-Sen Memorial Hospital

Jian-Zi Lin

Sun Yat-Sen Memorial Hospital

Man-Li Luo

Sun Yat-Sen Memorial Hospital

Lie Dai ( $\nabla$ dailie@mail.sysu.edu.cn )

Sun Yat-Sen Memorial Hospital, Sun Yat-Sen University

Research article

Keywords: Rheumatoid arthritis, Fibroblast-like synoviocytes, Artemether

Posted Date: June 16th, 2021

DOI: https://doi.org/10.21203/rs.3.rs-622553/v1

License: (c) (i) This work is licensed under a Creative Commons Attribution 4.0 International License.

Read Full License 


\section{Abstract}

Background: Fibroblast-like synoviocytes (FLS) in rheumatoid arthritis (RA) may cause articular damage as a result of its aggressive features including direct adhesion and invasion of surface cartilage in joints. Artemether (ART), one of the artemisinin derivatives with antimalarial properties, showed inhibitory effect on inflammation and destruction of joints in collagen-induced arthritis rats, which might be applied in RA treatment. However, whether ART has effects on the aggressive properties of human RA-FLS remains unexplored.

Methods: Synovium was obtained from patients with active RA $(n=18)$ and FLS were isolated in vitro. RAFLS were subjected to cell migration, invasion assays, live-cell imaging analysis and Rho GTPase activation assay after ART treatment. To identify the therapeutic target of ART, key signaling molecules of PI3K/Akt, AMPK, MAPK, NF-KB and mTOR pathways from RA-FLS were examined by Western Blot after ART treatment. Raptor was knockdown or overexpressed by siRNA or lentivirus transfection to reveal its role on regulating the aggressive properties of RA-FLS.

Results: ART treatment significantly suppressed the transwell migration and invasion of synovial FLS from RA patients. Time-lapse microscopy revealed that ART treatment reduced random migration velocity of RA-FLS, as well as the directional persistence. ART also impaired the formation of filopodia and lamellipodia in RA-FLS. Further mechanism investigation showed that ART reduced the protein level of Raptor, a critical component of the mTOR pathway, and its downstream target 4E-BP1. It also inhibited the activation of Rho GTPases and the expression of actin binding proteins, including Profilin 1 and pCofilin. Raptor overexpression could reverse the anti-migration and anti-invasion effects of ART on RAFLS as well as the suppression of Rho GTPases activation and the expression of actin binding proteins.

Conclusion: ART can inhibit migration of RA-FLS by blocking Raptor-induced actin polymerization. ART might be a potential agent targeting FLS in RA treatment.

\section{Introduction}

Rheumatoid arthritis (RA) is an aggressive immune-mediated joint disease with persistent synovitis and bone erosion, leading to disability and increased risk of mortality [1]. Systemic immune dysregulation and autoimmunity are considered as driving forces in the evolution of RA. However, though increasing number of drugs are available including the biologics and small molecules which target immune cells, cytokines or inflammatory signaling pathway, less than half of RA patients achieve ACR70 efficacy in clinical trials and there are still 10\%-15\% patients with refractory RA in real world practice [2]. On the other hand, current therapies for RA targeting the immune system are accompanied by potential risk of infection and malignancy. Therefore, new treatment options need to be developed especially effective and safe drugs with different targets for RA.

The main clinical manifestation of RA are synovitis and joint destruction which highlights the roles of stromal elements especially fibroblast-like synoviocytes (FLS) [3]. More and more evidences showed that 
RA-FLS not only strongly respond to an inflammatory environment, but also exhibit unique aggressive behaviors, actively and autonomously promote inflammation and directly adhere and invade to adjacent cartilage [4]. Benefiting from the new technologies, the characterization of specific cell types of RA synovium was analyzed in single-cell level, resulting in the identification of fibroblast subset with CD90+ which exhibited elevated migratory and invasive behavior $[5,6]$. In vivo study showed that injection of PDPN+FAPa+CD90+ fibroblast into the ankle of serum transfer induced arthritis mice resulted in more severe and sustained joint swelling with increased leukocyte infiltration [7]. The invasive behavior of RA-FLS is also positively associated with the rate of radiographic destruction of RA patients [8]. Recurrent or refractory RA might be due to the tumor-like phenotype of RA-FLS, and targeting FLS to restore articular homeostasis might be a potential option for RA therapy.

Methotrexate (MTX) is generally an anchor drug for RA treatment for its multiple mechanisms contributing to anti-inflammatory actions. But it also causes a variety of adverse events ranging from hair loss to fatal myelosuppression [9]. Antimalarials drugs are also suggested in RA management recommendation especially hydroxychloroquine (HCQ) which is mainly reserved for patients with mild RA because of its weak clinical efficacy and no joint protection [10]. Recent evidences showed that artemisinin and its synthetic derivatives had the potential therapeutic value for several autoimmune diseases including RA [11]. Artemether (ART), a peroxide sesquiterpenoid lipid-soluble derivative of artemisinin, is widely used in the treatment of Plasmodium falciparum and cerebral malaria [12]. It also exhibits a variety of potential anti-cancer, anti-inflammatory and anti-oxidant abilities [11, 13, 14]. More recently, a study showed that ART could inhibited RANKL-induced osteoclastogenesis in vitro via inhibition of MAPKs activation in bone marrow-derived macrophages isolated from mice [15]. These results implied that ART might be a new option for RA therapy. However, whether ART has effects on the invasive features of RA-FLS remains unexplored. Here, we aimed to explore whether ART had effects on the migration and invasion of RA-FLS as well as its regulation mechanism.

\section{Methods}

\section{Patients and FLS Preparation}

Synovium were obtained by Parker-Pearson needle biopsy on the knees of 18 active RA patients who fulfilled the 2010 classification criteria with clinical disease activity index (CDAl) $>2.8[16,17]$. There were 6 osteoarthritis $(\mathrm{OA})$ patients and 6 patients with non-inflammatory orthopedic arthropathies (Orth.A) including meniscus tear recruited as controls. Ethical approval was provided by the Research Ethics Committee of Sun Yat-sen Memorial Hospital (SYSEC-KY-KS-2019-072). Informed consent forms for all patients were signed.

FLS were isolated in vitro and identified by the light microscope and flow cytometry according to our previous described methods $[18,19]$. FLS was identified under the light microscope for morphology and further determined by the surface markers of CD90 and CD68 using flow cytometry. Primary FLS from passage 3 5 were used in further functional and molecular biological experiments. 


\section{Pharmacological Intervention on RA-FLS}

Primary RA-FLS were serum starvation for 6 hours before treated with corresponding concentrations of ART (Sigma-Aldrich, St. Louis, MO, USA) for indicated time, which was dissolved in dimethyl sulfoxide (DMSO, Sigma-Aldrich, St. Louis, MO, USA, less than $0.1 \% \mathrm{v} / \mathrm{v}$ ) and then diluted to working concentration with Dulbecco's Modified Eagle Medium (DMEM, Thermo Fisher Scientific, Waltham, MA, USA). MTX IC $5^{-}$ $10 \mathrm{nM}$ and $\mathrm{HCQ} \mathrm{IC}_{5}-20 \mu \mathrm{M}$ were used as control according to our previous study [19]. DMSO diluted with DMEM in equal volume was taken as untreated.

\section{Cell viability, Proliferation, Cell-cycle and Apoptosis Assays}

Primary RA-FLS were incubated with indicated concentrations of ART, MTX or HCQ for $6,12,24,36,48$ and 72 hours. The viable RA-FLS were quantified using the Cell Counting Kit-8 (CCK-8, Beyotime Biotechnology, Beijing, China). The proliferation of RA-FLS was detected by 5-ethynyl-2-deoxyuridine (EdU) staining using a Cell-Light EdU DNA Cell Proliferation Kit (RiboBio, Guangzhou, China) on 24 hours. The cell-cycle and apoptosis assays of RA-FLS were performed by flow cytometry on 24 hours [18, 19].

\section{ELISA Assay}

Primary RA-FLS were cultured in serum-free medium for 24 hours before incubated with $100 \mathrm{pg} / \mathrm{mL}$ tumor necrosis factor a (TNF-a, PeproTech, Rocky Hill, NJ, USA) and corresponding concentrations of ART, MTX or HCQ. ELISA assays were performed to detect IL-1 $\beta$, IL- 6 and IL-8 in the cell culture supernatants with a detecting absorbance of $450 \mathrm{~nm}$. Each plate test was repeated for three times.

\section{Cell Migration and Invasion Assays and Live-cell Imaging Analysis}

Primary RA-FLS were seeded at a density of $1 \times 10^{5}$ cells/well in six-well plates. After reaching a monolayer confluence over $90 \%$, RA-FLS were treated with Interventions of ART, MTX or HCQ at indicated concentrations were added to RA-FLS for 24 hours. Horizontal migration was detected by wound healing assay, while vertical migration and invasion were detected using transwell assays [19].

Primary RA-FLS were seeded at a density of $2 \times 10^{3}$ cells/well in 12-well plates and treated with indicated concentrations of ART, MTX or HCQ for 24 hours. Then live-cell imaging was performed on an inverted microscope Zeiss Axio Observer Z1 (Carl Zeiss, Berlin, Germany) using phase contrast illumination with a $10 \times(0.30 \mathrm{Ph} 1)$ objective at $37^{\circ} \mathrm{C}$ under $5 \% \mathrm{CO}_{2}$. For random migration, images were captured at 15 -min intervals and cells tracked using the Manual Tracking plugin for Image J software (NIH, Bethesda, Maryland, USA). In order to avoid errors caused by cells that divided or contacted with other cells during the experiment by the software analysis, we use manual methods for data analysis. Velocity was calculated as the total track distance divided by the total time (720 mins), and displacement rate (D/T) was calculated as the linear distance (D) divided by the total track distance $(T)$. Thirty cells from at least three independent experiments were analyzed. 


\section{Quantitative Real-time PCR Analysis (qPCR) and Western Blot Analysis}

Total RNA of primary FLS was extracted with Trizol (Takara, Kusatsu, Japan) and reverse-transcription was performed using PrimeScript ${ }^{\mathrm{TM}}$ RT reagent Kit (Takara, Kusatsu, Japan) [20]. The primer sequences of CDC42, Rac1, RhoA, Profilin 1, Cofilin, mTOR, Raptor, Rictor, GßL, 4E-BP1, p70S6K and GADPH were showed in Supplementary information table 1.

Western blot was performed to detect target protein expression from primary RA-FLS [21]. Primary antibodies recognizing PI3K, p-PI3K, Akt (pan), p-Akt (Thr308), AMPK-a, p-AMPK-a(Thr172), p38-MAPK, pp38 MAPK (Thr180/Tyr182) NF-kB p65, NF-kB p-p65 (Ser536), mTOR, p-mTOR (Ser2481), p-mTOR (Ser2448), Raptor, Rictor, GßL, 4E-BP1, p-4E-BP1, p70S6K, p-p70S6K, profilin 1, cofilin, p-cofilin (Ser3) and GADPH and secondary antibodies conjugated with HRP were obtained from Cell Signaling Technology (Danvers, MA, USA).

\section{Cells Transfection for Knockdown or Overexpression of Raptor}

Primary RA-FLS were transfected with Raptor-siRNA and negative control (Genepharma Co., Ltd, Shanghai, China). One of three independent designed siRNAs for Raptor knockdown was selected according to the transfection efficiency: 5'-GCUAAACAACAUGUCGCCATT-3', 5'-

UGGCGACAUGUUGUUUAGCTT-3. Transfections were performed using the Lipofectamine 3000 (Invitrogen, Carlsbad, CA, USA) according to the manufacturer's instructions.

Human full-length Raptor complementary DNA (CDNA) was cloned into lentiviral vector pLV [Exp] (named OE-Raptor), and empty pLV [Exp] vector alone was used as negative control (named OE-Vehicle). Whole cell lysates were extracted to assess transfection efficiency of Raptor in RA-FLS by qPCR and western blot.

\section{Rho GTPase Activation Assay}

GTP-bound RhoA, Rac1, and Cdc42 were measured using corresponding Activation Assay Kits (Cytoskeleton, Denver, CO, USA). The PAK-PBD affinity beads were incubated together with glutathioneagarose beads and then the complex (PAK-PBD/GTP) were recovered from the beads and analyzed using SDS-PAGE experiment as indicated by the manufacturer's protocol. Protein concentrations and activity forms of RhoA, Rac1, and Cdc42 in supernatants were assessed according to the manufacturer's instructions.

\section{Immunofluorescence Staining}

Primary RA-FLS were treated with different interventions for 24 hours and incubated with monoclonal rabbit anti-human Profilin antibody and mouse anti-human p-cofilin antibody (Cell Signaling Technology, Danvers, MA, USA) overnight at $4^{\circ} \mathrm{C}$ was followed by incubation with phalloidin-conjugated rhodamine (Beyotime, Bejing, China), Alexa Fluor ${ }^{\circledR}$ 488-labeled goat anti-rabbit IgG and Alexa Fluor ${ }^{\circledR}$ 647-labeled goat anti-mouse lgG secondary antibody for 60 mins in a dark wet box at $37^{\circ} \mathrm{C}$. Nucleus was stained with 
DAPI for 3 mins and coverslips were mounted with ProLong Gold Antifade Reagent (Invitrogen, Carlsbad, CA, USA). Images were analyzed using a Zeiss LSM 710 Confocal Imaging System.

\section{Statistical Analysis}

All statistical analyses were conducted using SPSS 21.0 statistical software and GraphPad 8.0 Software. Comparison between two groups was detected using Student's t-test. The data were presented as mean \pm SD or median and range for continuous variables and presented as frequencies and percentages for categorical variables and $P<0.05$ was considered significant [22].

\section{Results}

\section{ART Inhibited the Production of Cytokines from RA-FLS, but not the Cell Viability, Proliferation or Apoptosis}

Among 18 RA patients recruited in this study, their mean age was $49 \pm 8$ years with the median disease duration 64 months (range from 3 to 240 months), and the median CDAI 32 (range from 21 to 44). Among these patients, $78 \%$ of them had positive rheumatoid factor, $61 \%$ had positive anticyclic citrullinated peptide antibody. There were $22 \%$ patients had not received any glucocorticoids or disease modifying anti-rheumatic drugs (DMARDs) therapy for three months before recruitment.

Primary RA-FLS were cultured in vitro and identified with unique spindle morphology and high level of CD90 expression $(94.62 \% \pm 5.37 \%)$ but low level of CD68 expression $(1.40 \% \pm 0.75 \%)$ using flow cytometry (Figure S1). CCK-8 assay showed the $\mathrm{IC}_{50}$ of ART under 24 hours treatment on primary RA-FLS were up to $761 \mu \mathrm{M}$ (Figure S2A). RA-FLS exhibits tumor-like features including excessive proliferation and resistance of apoptosis. CCK-8, EdU and flow cytometry assays showed that ART IC ${ }_{5}-20 \mu \mathrm{M}$ as well as the controls of MTX IC $5_{5}-10 \mathrm{nM}$ or HCQ IC $5_{5}-20 \mu \mathrm{M}$ had no effect on cell viability, proliferation, cell-cycle or apoptosis of RA-FLS (all $P>0.05$, Figure S2B-D).

RA-FLS have also emerged as important immune modulator as they can secrete an abundance of proinflammatory cytokines, such as IL-1 $\beta$, IL- 6 and IL-8. Mimicking the inflammatory microenvironment of RA articular cavity by TNF-a $100 \mathrm{pg} / \mathrm{mL}$ stimulation for 24 hours, ART IC ${ }_{5}-20 \mu \mathrm{M}, \mathrm{MTX} \mathrm{IC}_{5}-10 \mathrm{nM}$ or HCQ $\mathrm{IC}_{5}-20 \mu \mathrm{M}$ significantly inhibited the level of IL-1 $\beta$, IL-6 and IL-8 by $18.2 \% \sim 63.1 \%$ reduction (all $P \otimes 0.05$, Figure S2E). These results showed that ART can inhibit the proinflammatory cytokines production of RAFLS at safety concentrations.

\section{ART Exhibited Anti-migration and Anti-invasion Effects on RA-FLS}

RA-FLS have the migratory potential by forming filopodia and lamellipodia, which leads to invasion of the pannus-cartilage interface of joints. To explore the effects of ART on RA-FLS migration and invasion, primary RA-FLS were pretreated with ART, MTX or HCQ at indicated concentrations for 24 hours. Compared with untreated RA-FLS, ART at $20 \mu \mathrm{M}$ significantly inhibited their migration distance in wound 
healing assay $(1063 \pm 84 \mu \mathrm{m}$ vs. $632 \pm 43 \mu \mathrm{m})$ and $38 \%$ less migrated cells in transwell migration assay at 12 hours ( $364 \pm 27$ cells/well vs. $226 \pm 21$ cells/well), as well as their invasion with $43 \%$ less invaded cells in transwell invasion assay at 24 hours $(312 \pm 50$ cells/well $v s .178 \pm 21$ cells/well, Figure 1$)$.

Tracing of migration tracks of sparsely seeded cells of RA-FLS by time-lapse microscopy, the results showed that ART at $20 \mu \mathrm{M}$ significantly inhibited random migration velocity $(0.294 \pm 0.099 \mu \mathrm{m} / \mathrm{min}$ vs. $0.386 \pm 0.104 \mu \mathrm{m} / \mathrm{min}, P<0.01)$, and the directional persistence with $36 \%$ decrease in displacement rate. Phalloidin staining images showed that ART at $20 \mu \mathrm{M}$ could impair the formation of filopodia and lamellipodia of RA-FLS with $45 \%$ reduction $(41.14 \% \pm 8.69 \%$ vs. $22.63 \% \pm 7.27 \%)$. MTX at $10 \mathrm{nM}$ showed similar anti-migration and anti-invasion effects on the RA-FLS as ART at $20 \mu \mathrm{M}$, whereas HCQ at $20 \mu \mathrm{M}$ showed no significant effects (Figure 1).

\section{ART Inhibited Rho Gtpases Activity and Actin-Binding Protein Expression in RA-FLS}

Rho GTPases including RhoA, Rac1, and Cdc42 mediate actin cytoskeleton organization in various cells. To validate whether ART inhibited the activation of Rho GTPases, qPCR, western blot and activation assay were performed after 24 hours treatment of ART, MTX or HCQ at indicated concentrations on primary RA-FLS. ART at $20 \mu \mathrm{M}$ significantly downregulated the mRNA and protein levels of RhoA, Rac1, and Cdc42 (Figure 2A). Cell lysate were assayed with $10 \mu \mathrm{g}$ of Rho binding domain beads. The amount of activated RhoA/Rac1/Cdc42 is determined by Western blot. ART at $20 \mu \mathrm{M}$ as well as MTX at 10 $\mathrm{nM}$ markedly inhibited the activation of RhoA, Rac1, and Cdc42, whereas HCQ at $20 \mu \mathrm{M}$ showed no significant effect on them (all $P<0.05$, Figure $2 A, B$ ).

Actin is the major component of the cytoskeleton regulating cell migration and invasion. Actin binding proteins including Profilin 1 and Cofilin are downstream targets of Rho GTPases, regulating actin polymerization and enable cell motility. To further explore whether ART inhibited the expression of actin binding proteins, qPCR and western blot were performed after 24 hours treatment of ART, MTX or HCQ at indicated concentrations on primary RA-FLS. ART at $20 \mu \mathrm{M}$ inhibited the protein level of Profilin 1 and p-Cofilin (Ser3), but not total Cofilin. MTX at $10 \mathrm{nM}$ significantly inhibited all actin binding proteins, whereas HCQ at $20 \mu \mathrm{M}$ showed no significant effect on them (Figure $2 \mathrm{C}$ ).

\section{ART Inhibited the mTOR Pathway Activation in RA-FLS}

There are several signaling pathways usually involved in RA-FLS which might be the upstream regulators of the Rho GTPases, including MAPK, NF-KB and mTOR pathways which are associated with inflammation, and integrin/galectin triggered PI3K/Akt pathway, modulator of energy balance AMPK pathway [23-25]. In order to identify the therapeutic target of ART, we examined the key signaling molecules of PI3K/Akt (PI3K, p-PI3K, Akt and p-Akt), AMPK (AMPK-a and p-AMPK-a), MAPK (p38-MAPK and p-p38 MAPK), NF-kB (NF-kB p65 and NF-kB p-p65) and mTOR (mTOR, p-mTOR, Raptor, Rictor and $\mathrm{G} \beta L$ ) pathways after treatment with $A R T, M T X$ or HCQ at indicated concentrations on primary RA-FLS for 24 hours. ART at $20 \mu \mathrm{M}$ significantly inhibited Raptor expression, whereas it did not alter the expression 
of signaling molecules from other pathways. MTX at $10 \mathrm{nM}$ significantly inhibited Raptor and NF-KB pp65, whereas HCQ at $20 \mu \mathrm{M}$ had no effect on these pathways (Figure 3A, B and Figure S3).

We further investigated whether ART had effect on the activation of the mTOR pathway components. Raptor and Rictor are the key proteins of mTORC1 and mTORC2, respectively. We found that ART at $20 \mu \mathrm{M}$ significantly inhibited the expression of Raptor but not Rictor (Figure 3C). 4E-BP1 and p70S6K1 are the major substrates of Raptor. After treatment with ART, MTX or HCQ at indicated concentrations on primary RA-FLS for 24 hours, western blot showed that ART at $20 \mu \mathrm{M}$ significantly inhibited the expression and the phosphorylation of 4E-BP1, but not p70S6K, which implied that ART might inhibit the activation of mTOR pathway through suppressing Raptor and 4E-BP1 expression. MTX at $10 \mathrm{nM}$ significantly inhibited Raptor and its downstream regulators of 4E-BP1, p-4E-BP1 and p70S6K, whereas HCQ at $20 \mu \mathrm{M}$ had no effect on them (Figure 3D).

\section{Elevated Raptor in RA-FLS Promoted Migration and Invasion with Actin Cytoskeleton Regulation}

Little was known about Raptor expression in RA-FLS as well as its function. The results of GPCR and western blot showed that Raptor prominently up-regulated over 2.2-fold in RA-FLS in MRNA and protein levels when compared with that in OA-FLS or Orth.A-FLS, respectively (both $P<0.05$, Figure 4A). Raptor knockdown in RA-FLS by siRNA transfection significantly suppressed their migration and invasion, as well as the Rho GTPase activity and the expression of Profilin 1 and p-Cofilin (Ser3). Conversely, Raptor overexpression by lentivirus infection had opposite effects on RA-FLS (Figure 4B D). Immunofluorescence staining of Profilin 1, p-Cofilin (Ser3) and F-actin in RA-FLS showed that Raptor knockdown in RA-FLS led to a reduction in lamellipodia, filopodia and actin stress formation, whereas Raptor overexpression promoted their formation (Figure 5). These results implied that elevated Raptor in RA-FLS can promote the aggressive properties, activate Rho GTPase and regulate actin cytoskeleton.

\section{ART Exhibited Anti-migration and Anti-invasion Effects on RA-FLS via Inhibiting Raptor and Actin Polymerization}

Compared with Raptor knockdown, ART at $20 \mu \mathrm{M}$ had similar anti-migration and anti-invasion effects on RA-FLS as well as the Rho GTPase activity and the expression of Profilin 1 and p-Cofilin (Ser3). To verify whether ART suppresses the migration and invasion of RA-FLS via inhibiting Raptor and actin polymerization, primary RA-FLS were transfected with lentivirus for Raptor overexpression. After treatment with ART at $20 \mu \mathrm{M}$ for 24 hours, Raptor overexpression could reverse the anti-migration and anti-invasion effects of ART on RA-FLS as well as the activity of Rho GTPases and the Profilin 1 and pcofilin (Ser3) expression (Figure 4 B D). Immunofluorescence staining showed that up-regulated Raptor could also reverse the suppression of the formation of lamellipodia, filopodia and stress fibers by ART in RA-FLS (Figure 5). These results indicated that ART can inhibit actin polymerization of RA-FLS via Raptor pathway and resulted in the suppression on migration and invasion of RA-FLS.

\section{Discussion}


In the present study, we first report that ART has anti-migration and anti-invasion as well as antiinflammatory effects on RA-FLS, which are similar to MTX but greater than HCQ. We further find a novel role of Raptor, a critical regulator among mTOR pathway, on promoting migration and invasion of primary RA-FLS via activating Rho GTPase and regulating actin cytoskeleton, while ART can block the Raptor/4EBP1/Profilin 1 pathway and regulate actin cytoskeleton to exert its inhibitory effects (Figure 6). Our study provides experimental evidence of the potential therapeutic value of ART on targeting FLS in RA treatment and reveals its therapeutic target of Raptor on regulating the aggressive properties of RA-FLS.

The antimalarial effects of artemisinin and its derivatives base on the peroxide bridges which will be broken into the nucleophilic radical metabolites by the heme or free iron especially in parasite-infected red blood cells. Then the free radicals acting as alkylating agents, leading to parasitic death [26]. Previous studies showed artesunate, one of the artemisinin derivatives, suppressed IL-1 $1 \beta, \mathrm{IL}-6, \mathrm{IL}-8$ and VEGF expression by inhibition of the key inflammatory signaling such as NF-KB, PI3K/Akt or MAPK pathways [27-29]. More recently we reported that artesunate could inhibit the migration and invasion of RA-FLS, implying the potential therapeutic value of artemisinin derivatives for RA. ${ }^{19}$ ART is a lipid-based derivative and has a longer half-life of 1.6 hours than that of water-soluble artesunate as 0.3 hours according to the labels from FDA [30]. Here, we find that ART has anti-migration and anti-invasion effects on RA-FLS, suggesting that ART might be another efficient agent targeting FLS in RA.

We further investigated the mechanism of ART on inhibiting RA-FLS migration and invasion. RhoA, Rac1, and Cdc42 are the best characterized members of Rho GTPases. RhoA initiates the formation of contractile actin, as well as stress fibers, while Rac1 and Cdc42 can promote the lamellipodia and filopodia formation, respectively [31]. Rho GTPases are reported to play critical roles on the aggressive properties of RA-FLS which majorly regulate their migration [32,33]. The actin binding proteins including Profilin 1 and Cofilin are downstream targets of Rho GTPases which regulate actin polymerization and enable cell motility. Cofilin mediates the actin filaments disassembly but the cofilin (Ser3) phosphorylation inactivates its function, while Profilin 1 plays a critical role on actin polymerization [34]. In our study, we find that ART significantly inhibits the total mRNA and protein expression of Rho GTPases and their activation, as well as the actin binding proteins of Profilin 1 and $p$ cofilin (Ser3), which clarify the role of ART on suppressing RA-FLS migration and the following invasion by blocking the actin polymerization.

The mTOR is a serine/threonine kinase regulating various cell functions such as cell proliferation and survival under the stimulation of cytokines and hormones [35]. It is composed of two multi-protein complexes, which are termed mTORC1 and mTORC2. The mTORC1 is involved in mRNA translation, protein turnover and metabolism, while mTORC2 regulates proliferation and survival [36]. Raptor promotes the recruitment of mTOR substrates and is necessary for MTORC1 to achieve subcellular localization [37]. 4E-BP1 is one of the signature substrates phosphorylated by Raptor and regulates protein synthesis of RhoA, Rac1, and Cdc42 [38]. Inhibition of mTOR reduced synovitis as well as articular cartilage and bone destruction in human TNF-transgenic mice [39]. Rapamycin inhibited lamellipodia formation and RA-FLS invasion by suppressing the phosphorylation of mTOR and its substrates 
including p70S6K1 and 4E-BP1 [40]. Recent in vitro study showed that IL-17-induced L-type Amino Acid Transporter 1 overexpression might enhance the migration of RA-FLS via the mTOR/4E-BP1 pathway [41]. In this study, we find that Raptor is the therapeutic target of ART and verified that Raptor is the critical component of $\mathrm{mTORC} 1$ on promoting migration and invasion and regulating actin cytoskeleton.

We also compared the efficacy of ART with MTX or HCQ at the same safety concentrations. MTX has anti-inflammatory effect on RA-FLS by stimulating adenosine and its receptor, leading to inhibition of NFKB activity [9]. However, MTX might also cause toxic effects including stomatitis, nausea, and bone marrow depression because of its antifolate property, so it is used in a low dose in clinical practice. Previous study on collagen-induced arthritis rats showed that compared with intraperitoneally injection of MTX, intramuscularly administration of ART ( $3 \mathrm{mg} / \mathrm{kg} / \mathrm{day}$ ) similarly reduced inflammation and bone erosion in the paws with greater tolerability [42]. In another study, intraperitoneal injection of ART (10 mg/kg/day) showed anti-fibrosis effect in hepatic fibrosis mice model [43]. HCQ has antiinflammatory effect on lymphocytes by inhibiting lysosomal and autophagosome functions [44]. Intervention of HCQ on RA-FLS is rarely reported. Our previous study showed that HCQ could inhibit IL-1 $\beta$, IL- 6 and IL-8 production, but not the migration and invasion of RA-FLS [19]. In the present research, we find that ART has anti-migration and anti-invasion as well as anti-inflammatory effects on RA-FLS which is similarly to MTX and superior to HCQ. The effective dose of ART in our study was $20 \mu \mathrm{M}$ (approximately equivalent to $5.96 \mathrm{mg} / \mathrm{kg}$ ), which was higher than the dose of previous animal study but was in the safety range of no more than $40 \mathrm{mg} / \mathrm{kg}[30,42]$. Though the working dose of ART is obviously higher than MTX in vitro study, the dose of ART ( $3 \mathrm{mg} / \mathrm{kg} /$ day) reduces to about 23 times of that of MTX ( $0.3 \mathrm{mg} / \mathrm{kg}$ for 3 times weekly) in the treatment of collagen-induced arthritis rats [42]. It is applicable to obtain the effective dose in our study in vivo through systemic or localized administration and ART might be a safety and effective medicine for RA therapy.

In conclusion, our study first reports the novel anti-migration and anti-invasion effects of ART on RA-FLS, and illuminates its mechanism of blocking Raptor-induced actin polymerization and therefore suppressing lamellipodia, filopodia and actin stress formation. ART might be a potential agent targeting FLS in RA.

\section{Abbreviations}

ACR: American College of Rheumatology; ART: Artemether; CCK-8: Cell counting kit-8; CDAl: Clinical disease activity index; DMARDs: Disease-modifying anti-rheumatic drugs; DMEM: Dulbecco's Modified Eagle Medium; DMSO: Dissolved in dimethyl sulfoxide; FLS: Fibroblast-like synoviocytes; HCQ:

Hydroxychloroquine; MMP: Matrix metalloproteinase; MTX: Methotrexate; OA: Osteoarthritis; Orth.A: Orthopedic arthropathies; RA: Rheumatoid arthritis; TNF-a: Tumor necrosis factor a

\section{Declarations}


Acknowledgements: We thank all subjects and medical staff who generously contributed to this study.

Author contributions $\square A l l$ authors contributed to the final manuscript. MJD and JJ contributed equally to this work, conceived and designed the study, performed the experiments and statistical analysis, and drafted the manuscript; Corresponding author DL and LML conceived and participated in its design, advised on the research, analysis of results, and critically revised the manuscript. WJW, ZXP, LQH, MYQ and LJZ participated in performing the experiments and revised the manuscript. All authors critically revised the manuscript for important intellectual content and approved its final version.

Funding: This work was supported by National Natural Science Foundation of China (No. 81801606, 81971527 and 82001731), Natural Science Foundation of Guangdong Province (No. 2017 A030310236 and 2018A030313541), Guangdong Basic and Applied Basic Research Foundation (No. 2019A1515011928 and 2019A1515110125), Science and Technology Program of Guangzhou (No. 201904010088 and 202102010188) and Yat-Sen Scholarship for Young Scientist.

\section{Availability of data and materials:}

The datasets used and/or analyzed during the current study are available from the corresponding author on reasonable request.

Ethics approval and consent to participate: This study was conducted in compliance with the Helsinki Declaration. The Medical Ethics Committee of Sun Yat-sen Memorial Hospital approved the protocol (SYSEC-KY-KS-2019-072). All patients agreed to participate in this study and signed written informed consent.

Consent for publication: All participants have approved to publish the data in this manuscript.

Competing interests: All authors have read the policy on the disclosure of potential conflicts and have no competing interests to declare.

\section{Author details:}

${ }^{1}$ Department of Rheumatology, Sun Yat-Sen Memorial Hospital, Sun Yat-Sen University, Guangzhou, P.R China

${ }^{2}$ Guangdong Provincial Key Laboratory of Malignant Tumor Epigenetics and Gene Regulation, Sun YatSen Memorial Hospital, Sun Yat-Sen University, Guangzhou China

${ }^{3}$ Medical Research Center, Sun Yat-Sen Memorial Hospital, Sun Yat-Sen University, Guangzhou, China

\section{References}

1. Sparks JA. Rheumatoid Arthritis. Ann Intern Med. 2019; 170(1): ITC1-ITC16. 
2. Winthrop KL, Weinblatt ME, Bathon J, Burmester GR, Mease PJ, Crofford L, et al. Unmet need in rheumatology: reports from the Targeted Therapies meeting 2019. Ann Rheum Dis. 2020; 79(1):8893.

3. You S, Koh JH, Leng L, Kim WU, Bucala R. The Tumor-Like Phenotype of Rheumatoid Synovium: Molecular Profiling and Prospects for Precision Medicine. Arthritis Rheumatol. 2018; 70(5):637-52.

4. Bustamante MF, Garcia-Carbonell R, Whisenant KD, Guma M. Fibroblast-like synoviocyte metabolism in the pathogenesis of rheumatoid arthritis. Arthritis Res Ther. 2017; 19(1):110.

5. Mizoguchi F, Slowikowski K, Wei K, Marshall JL, Rao DA, Chang SK, et al. Functionally distinct disease-associated fibroblast subsets in rheumatoid arthritis. Nat Commun. 2018; 9(1):789.

6. Stephenson W, Donlin LT, Butler A, Rozo C, Bracken B, Rashidfarrokhi A, et al. Single-cell RNA-seq of rheumatoid arthritis synovial tissue using low-cost microfluidic instrumentation. Nat Commun. 2018; 9(1):791.

7. Croft AP, Campos J, Jansen K, Turner JD, Marshall J, Attar M, et al. Distinct fibroblast subsets drive inflammation and damage in arthritis. Nature. 2019; 570(7760):246-51.

8. Tolboom TC, van der Helm-Van MA, Nelissen RG, Breedveld FC, Toes RE, Huizinga TW. Invasiveness of fibroblast-like synoviocytes is an individual patient characteristic associated with the rate of joint destruction in patients with rheumatoid arthritis. Arthritis Rheum. 2005; 52(7):1999-2002.

9. Cronstein BN, Aune TM. Methotrexate and its mechanisms of action in inflammatory arthritis. Nat Rev Rheumatol. 2020; 16(3):145-54.

10. Smolen JS, Landewe R, Bijlsma J, Burmester GR, Dougados M, Kerschbaumer A, et al. EULAR recommendations for the management of rheumatoid arthritis with synthetic and biological diseasemodifying antirheumatic drugs: 2019 update. Ann Rheum Dis. 2020; 79(6):685-99.

11. An J, Minie M, Sasaki T, Woodward JJ, Elkon KB. Antimalarial Drugs as Immune Modulators: New Mechanisms for Old Drugs. Annu Rev Med. 2017; 68:317-30.

12. Organization, W. H. Guidelines for the Treatment of Malaria. 3rd ed. Geneva: WHO Press, 2015.

13. Wong YK, Xu C, Kalesh KA, He Y, Lin Q, Wong W, et al. Artemisinin as an anticancer drug: Recent advances in target profiling and mechanisms of action. Med Res Rev. 2017; 37(6):1492-517.

14. Li S, Zhao X, Lazarovici P, Zheng W. Artemether Activation of AMPK/GSK3beta(ser9)/Nrf2 Signaling Confers Neuroprotection towards beta-Amyloid-Induced Neurotoxicity in 3xTg Alzheimer's Mouse Model. Oxid Med Cell Longev. 2019; 2019:1862437.

15. Wu H, Hu B, Zhou X, Zhou C, Meng J, Yang Y, et al. Artemether attenuates LPS-induced inflammatory bone loss by inhibiting osteoclastogenesis and bone resorption via suppression of MAPK signaling pathway. Cell Death Dis. 2018; 9(5):498.

16. Schumacher HJ, Kulka JP. Needle biopsy of the synovial membrane--experience with the ParkerPearson technic. N Engl J Med. 1972; 286(8):416-9.

17. Aletaha D, Neogi T, Silman AJ, Funovits J, Felson DT, Bingham CR, et al. 2010 Rheumatoid arthritis classification criteria: an American College of Rheumatology/European League Against Rheumatism 
collaborative initiative. Arthritis Rheum. 2010; 62(9):2569-81.

18. Zhou JJ, Ma JD, Mo YQ, Zheng DH, Chen LF, Wei XN, et al. Down-regulating peroxisome proliferatoractivated receptor-gamma coactivator-1 beta alleviates the proinflammatory effect of rheumatoid arthritis fibroblast-like synoviocytes through inhibiting extracellular signal-regulated kinase, p38 and nuclear factor-kappaB activation. Arthritis Res Ther. 2014; 16(5):472.

19. Ma JD, Jing J, Wang JW, Yan T, Li QH, Mo YQ, et al. A novel function of artesunate on inhibiting migration and invasion of fibroblast-like synoviocytes from rheumatoid arthritis patients. Arthritis Res Ther. 2019; 21(1):153.

20. Ma JD, Jing J, Wang JW, Mo YQ, Li QH, Lin JZ, et al. Activation of the Peroxisome ProliferatorActivated Receptor gamma Coactivator 1beta/NFATc1 Pathway in Circulating Osteoclast Precursors Associated With Bone Destruction in Rheumatoid Arthritis. Arthritis Rheumatol. 2019; 71(8):1252-64.

21. Zhou Q, Chen X, He H, Peng S, Zhang Y, Zhang J, et al. WD repeat domain 5 promotes chemoresistance and Programmed Death-Ligand 1 expression in prostate cancer. Theranostics. $2021 ; 11(10): 4809-24$.

22. Chen X, Zhang J, Ruan W, Huang M, Wang C, Wang H, et al. Urine DNA methylation assay enables early detection and recurrence monitoring for bladder cancer. J Clin Invest. 2020; 130(12):6278-89.

23. Shi M, Wang J, Xiao Y, Wang C, Qiu Q, Lao M, et al. Glycogen Metabolism and Rheumatoid Arthritis: The Role of Glycogen Synthase 1 in Regulation of Synovial Inflammation via Blocking AMPActivated Protein Kinase Activation. Front Immunol. 2018; 9:1714.

24. Karonitsch T, Kandasamy RK, Kartnig F, Herdy B, Dalwigk K, Niederreiter B, et al. mTOR Senses Environmental Cues to Shape the Fibroblast-like Synoviocyte Response to Inflammation. Cell Rep. 2018; 23(7):2157-2167.

25. Yoshitomi H. Regulation of Immune Responses and Chronic Inflammation by Fibroblast-Like Synoviocytes. Front Immunol. 2019; 10:1395.

26. Hou L, Huang H. Immune suppressive properties of artemisinin family drugs. Pharmacol Ther. 2016; 166:123-7.

27. Shi C, Li H, Yang Y, Hou L. Anti-inflammatory and immunoregulatory functions of artemisinin and its derivatives. Mediators Inflamm. 2015; 2015:435713.

28. Xu H, He Y, Yang X, Liang L, Zhan Z, Ye Y, et al. Anti-malarial agent artesunate inhibits TNF-alphainduced production of proinflammatory cytokines via inhibition of NF-kappaB and PI3 kinase/Akt signal pathway in human rheumatoid arthritis fibroblast-like synoviocytes. Rheumatology (Oxford). 2007; 46(6):920-6.

29. He Y, Fan J, Lin H, Yang X, Ye Y, Liang L, et al. The anti-malaria agent artesunate inhibits expression of vascular endothelial growth factor and hypoxia-inducible factor-1alpha in human rheumatoid arthritis fibroblast-like synoviocyte. Rheumatol Int. 2011; 31(1):53-60.

30. Drugs@FDA: FDA-Approved Drugs. https://www.accessdata.fda.gov/scripts/cder/daf/index.cfm

31. Warner H, Wilson BJ, Caswell PT. Control of adhesion and protrusion in cell migration by Rho GTPases. Curr Opin Cell Biol. 2019; 56:64-70. 
32. Peng WX, Zhu SL, Zhang BY, Shi YM, Feng XX, Liu F, et al. Smoothened Regulates Migration of Fibroblast-Like Synoviocytes in Rheumatoid Arthritis via Activation of Rho GTPase Signaling. Front Immunol. 2017; 8:159.

33. Pollard TD. Actin and Actin-Binding Proteins. Cold Spring Harb Perspect Biol. 2016; 8(8):a018226.

34. Zou Y, Xu S, Xiao Y, Qiu Q, Shi M, Wang J, et al. Long noncoding RNA LERFS negatively regulates rheumatoid synovial aggression and proliferation. J Clin Invest. 2018; 128(10):4510-24.

35. Perl A. Activation of mTOR (mechanistic target of rapamycin) in rheumatic diseases. Nat Rev Rheumatol. 2016; 12(3):169-82.

36. Saxton RA, Sabatini DM. mTOR Signaling in Growth, Metabolism, and Disease. Cell. 2017; 168(6):960-76.

37. Suto T, Karonitsch T. The immunobiology of mTOR in autoimmunity. J Autoimmun. 2020; 110:102373.

38. Liu L, Luo Y, Chen L, Shen T, Xu B, Chen W, et al. Rapamycin inhibits cytoskeleton reorganization and cell motility by suppressing RhoA expression and activity. J Biol Chem. 2010; 285(49):38362-73.

39. Cejka D, Hayer S, Niederreiter B, Sieghart W, Fuereder T, Zwerina J, et al. Mammalian target of rapamycin signaling is crucial for joint destruction in experimental arthritis and is activated in osteoclasts from patients with rheumatoid arthritis. Arthritis Rheum. 2010; 62(8):2294-302.

40. Laragione T, Gulko PS. mTOR regulates the invasive properties of synovial fibroblasts in rheumatoid arthritis. Mol Med. 2010; 16(9-10):352-8.

41. Yu Z, Lin W, Rui Z, Jihong P. Fibroblast-like synoviocyte migration is enhanced by IL-17-mediated overexpression of L-type amino acid transporter 1 (LAT1) via the mTOR/4E-BP1 pathway. Amino Acids. 2018; 50(2):331-40.

42. Cuzzocrea S, Saadat F, Di Paola R, Mirshafiey A. Artemether: a new therapeutic strategy in experimental rheumatoid arthritis. Immunopharmacol Immunotoxicol. 2005; 27(4):615-30.

43. Li Y, Jin C, Shen M, Wang Z, Tan S, Chen A, et al. Iron regulatory protein 2 is required for artemether mediated anti-hepatic fibrosis through ferroptosis pathway. Free Radic Biol Med. 2020; 160:845-59.

44. Schrezenmeier E, Dorner T. Mechanisms of action of hydroxychloroquine and chloroquine: implications for rheumatology. Nat Rev Rheumatol. 2020; 16(3):155-66.

\section{Figures}


A

Untreated
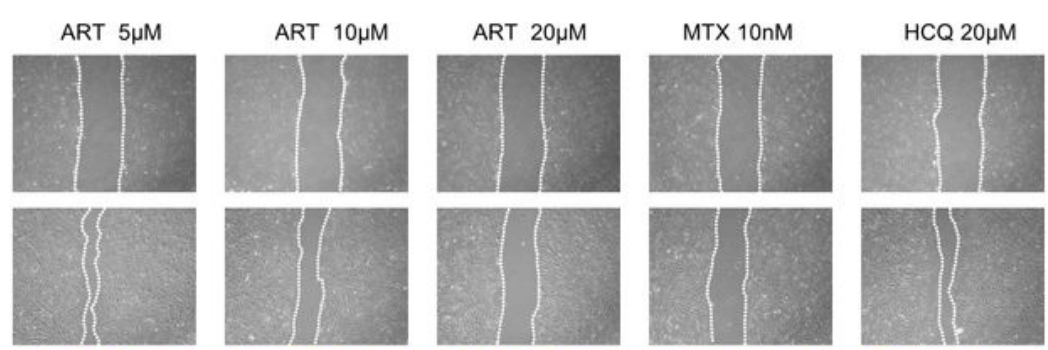

B
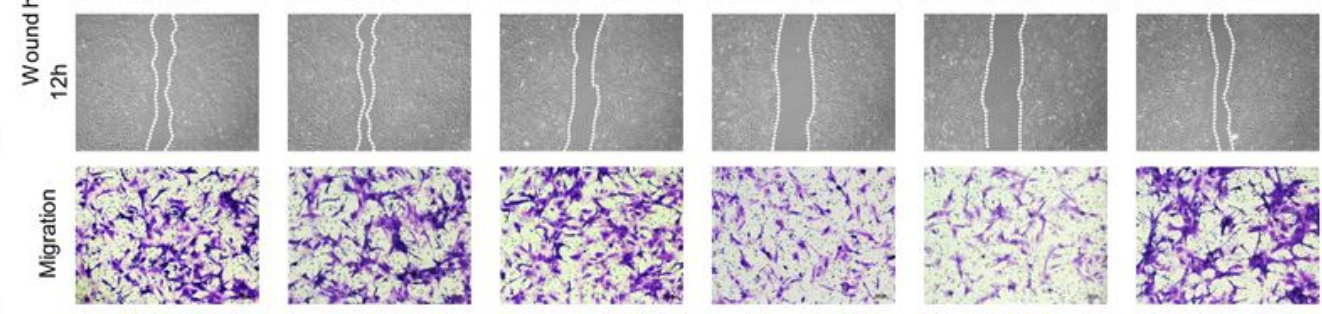

C
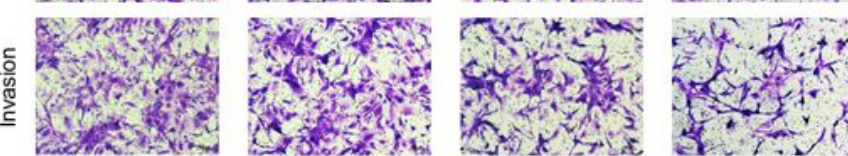

D
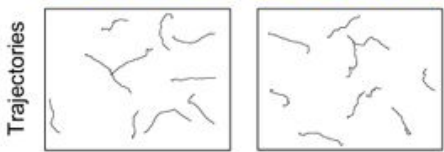

$\mathbf{E}$
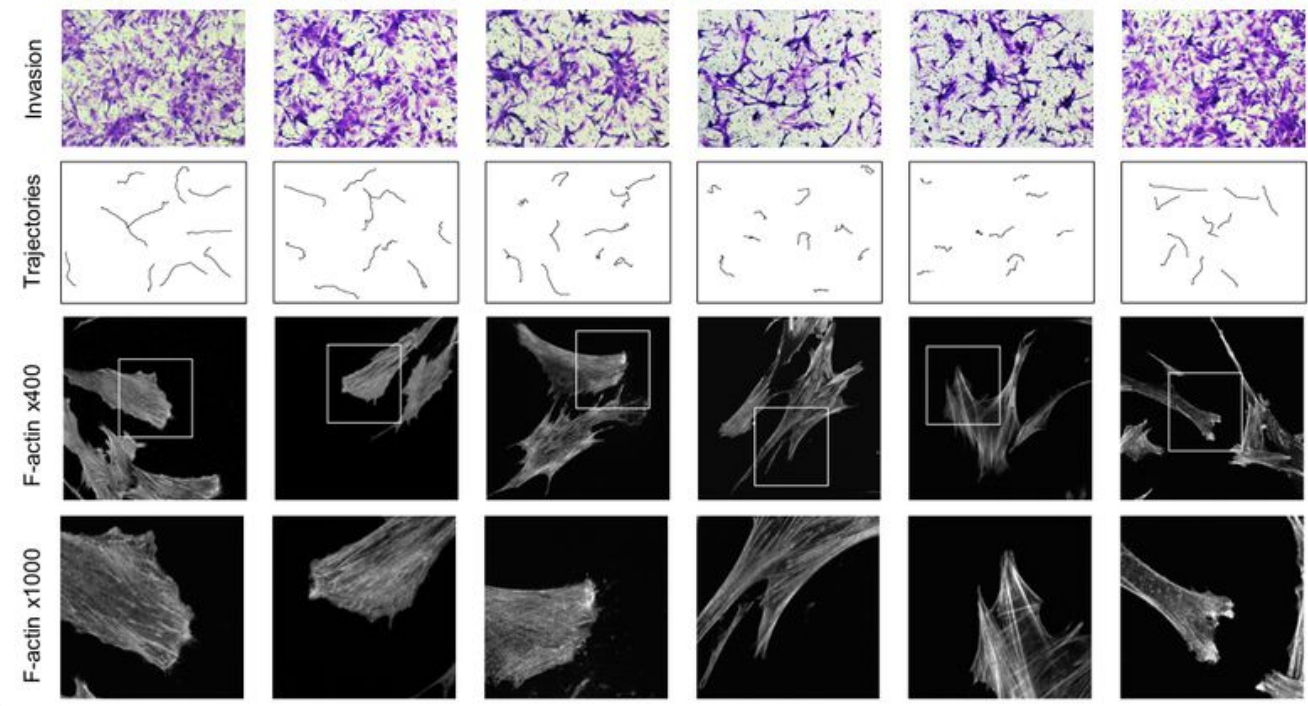

$\mathbf{F}$
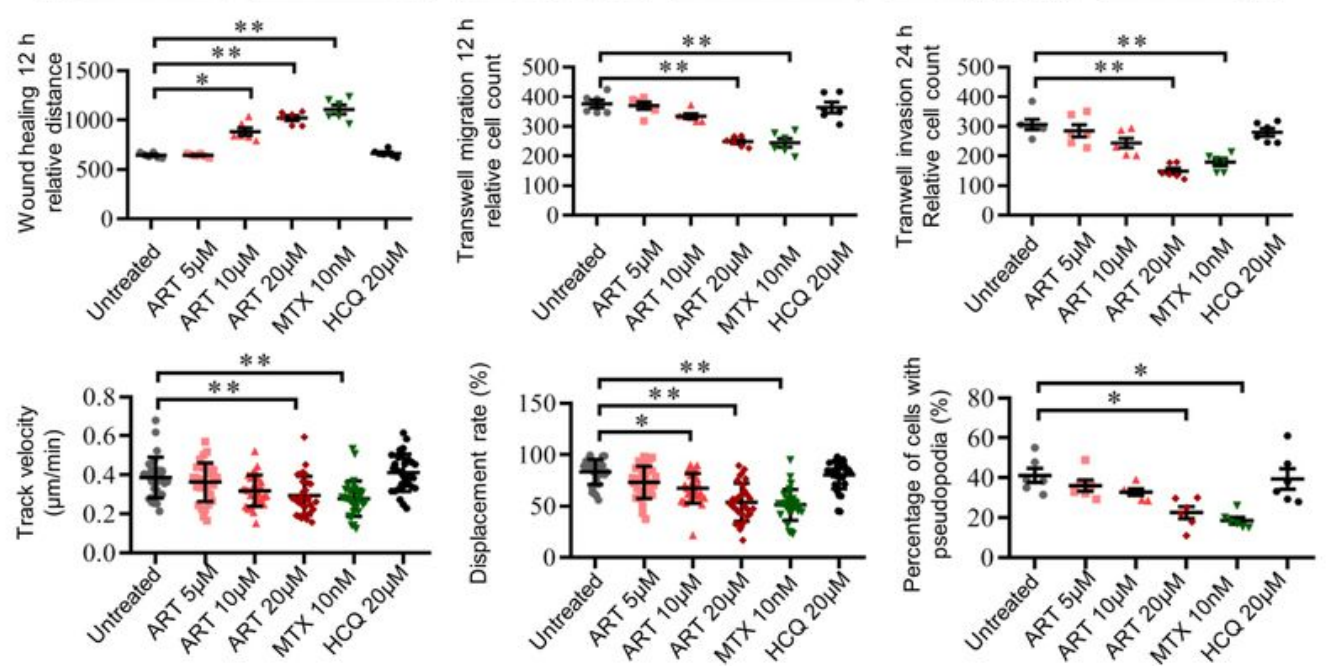

\section{Figure 1}

Effects of ART on migration and invasion of RA-FLS. Primary RA-FLS were treated with ART, MTX or HCQ for 24 hours. (A C) Wound healing and transwell assays showed the capacity of horizontal migration (A), vertical migration (B) and invasion (C). (D) Trajectories of randomly migrating cells were tracked by timelapse microscopy over a 12-h period and 10 trajectories of representative cells were shown. (E) F-actin was stained with phalloidin. Representative images were shown (original magnification, $\times 400$ above and 
$\times 1000$ below). (F) Statistics for the effects of ART, MTX or HCQ on RA-FLS migration and invasion. Data were represented as means \pm SD from 6 RA patients. ${ }^{*} P<0.05$, ${ }^{\star *} P<0.01$, ${ }^{\star *} * P<0.001$, compared with untreated (RA-FLS treated with DMSO). ART: artemether, MTX: methotrexate, HCQ: hydroxychloroquine.

A
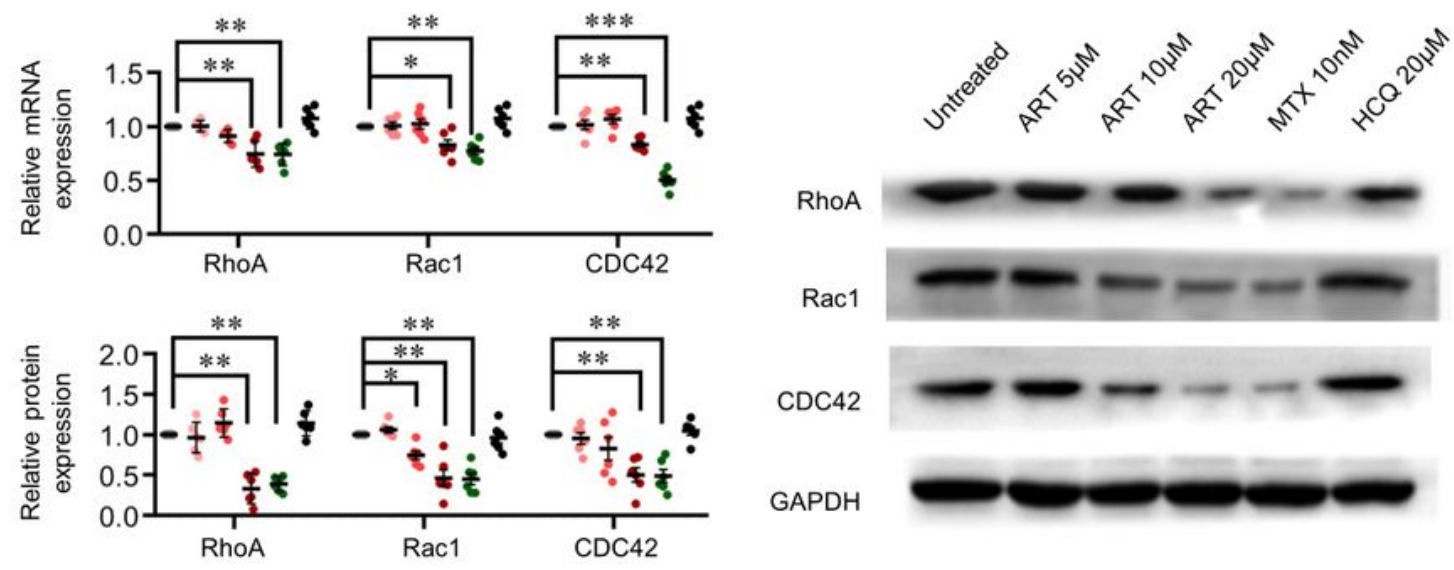

B
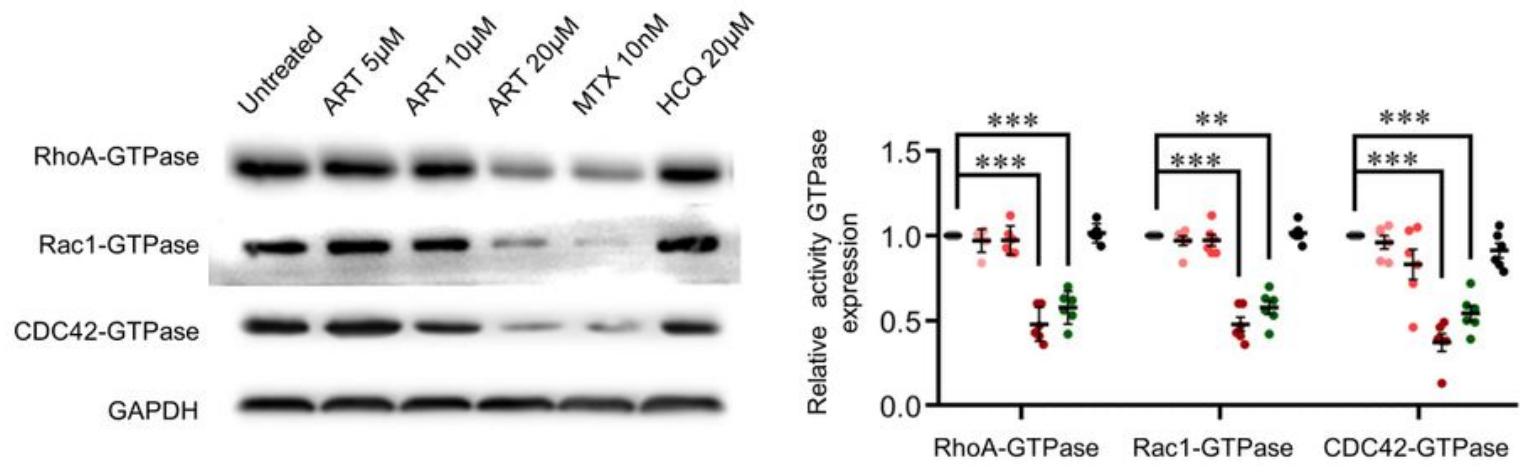

C
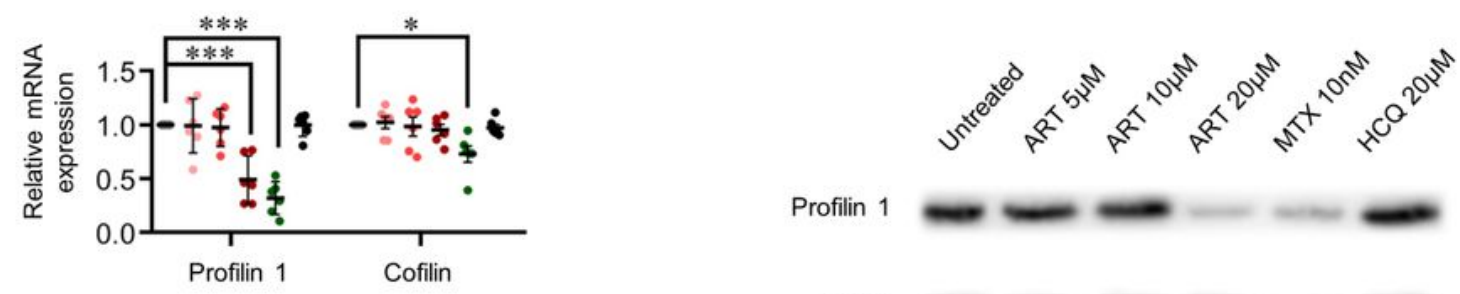

Profilin 1
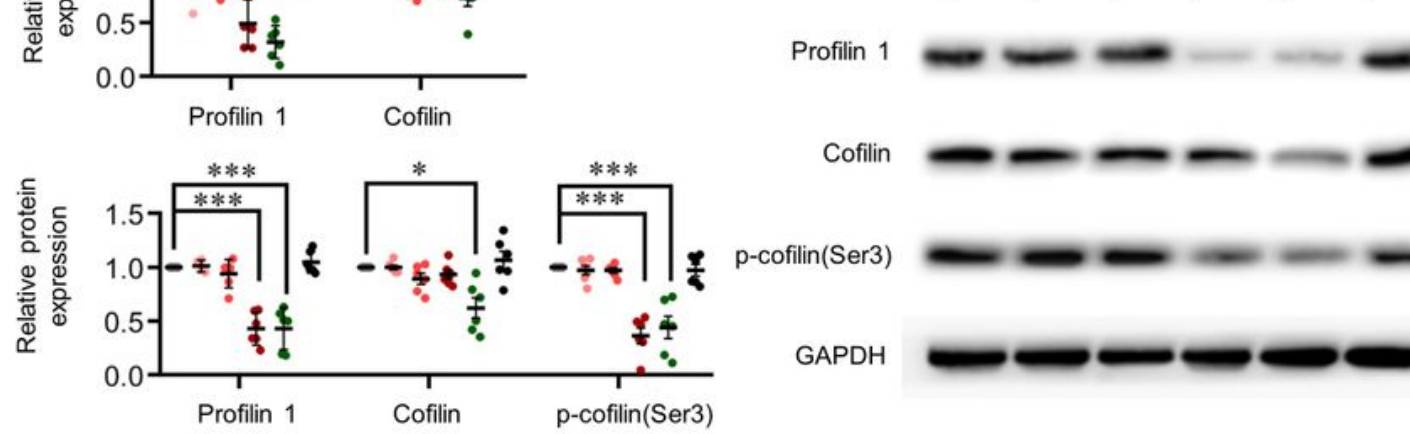

p-cofilin(Ser3)

GAPDH

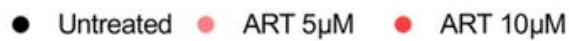

- ART $20 \mu \mathrm{M}$

MTX 10nM

$\mathrm{HCQ} 20 \mu \mathrm{M}$

Figure 2

Effects of ART on Rho-GTPases and actin-binding proteins in RA-FLS. Primary RA-FLS were treated with ART, MTX or HCQ for 24 hours. (A) Total mRNA and protein expression of RhoA, Rac1, Cdc42 were 
analyzed by qPCR and western blot. (B) Activity of RhoA, Rac1 and Cdc42 were measured by RhoA/Rac1/Cdc42 activation assay. (C) Total mRNA and protein expression of Profilin 1, Cofilin and pcofilin (Ser3) were analyzed by qPCR and western blot. Relative protein expression was normalized by GAPDH. Data were represented as means \pm SD from 6 RA patients. ${ }^{*} P<0.05,{ }^{*} P<0.01,{ }^{*} *{ }^{*} P<0.001$, compared with untreated (RA-FLS treated with DMSO). ART: artemether, MTX: methotrexate, HCQ: hydroxychloroquine.

A

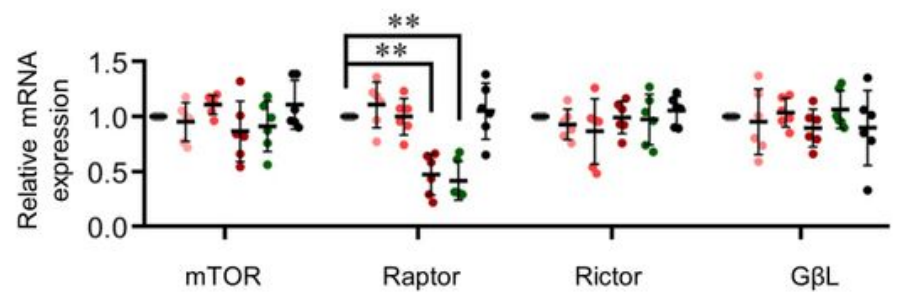

C

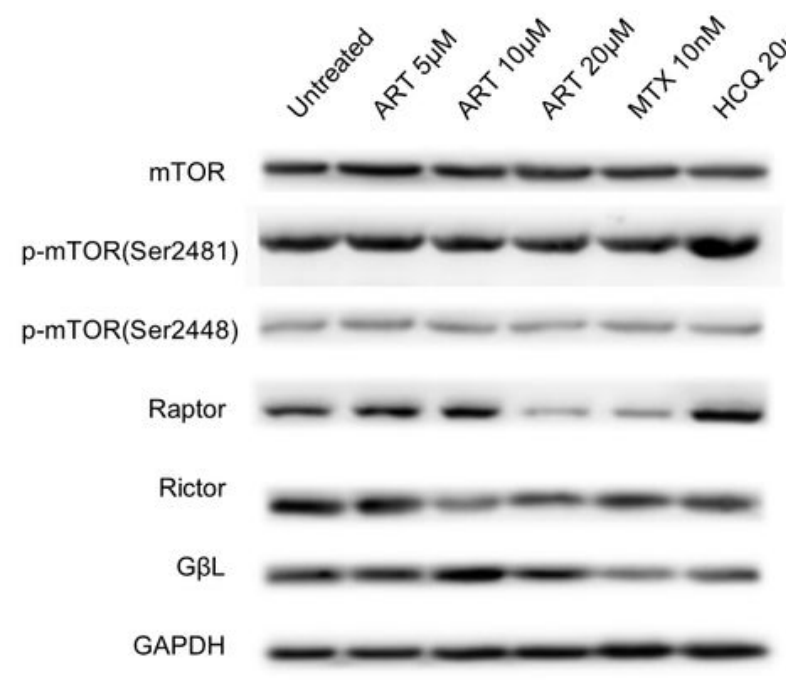

B
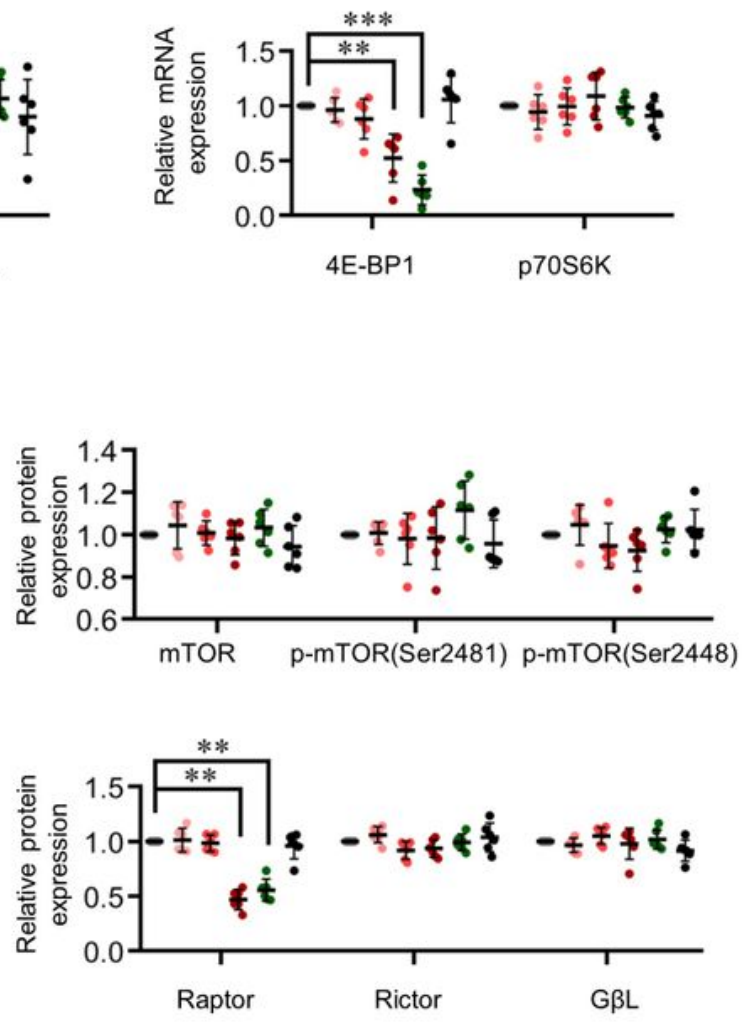

D
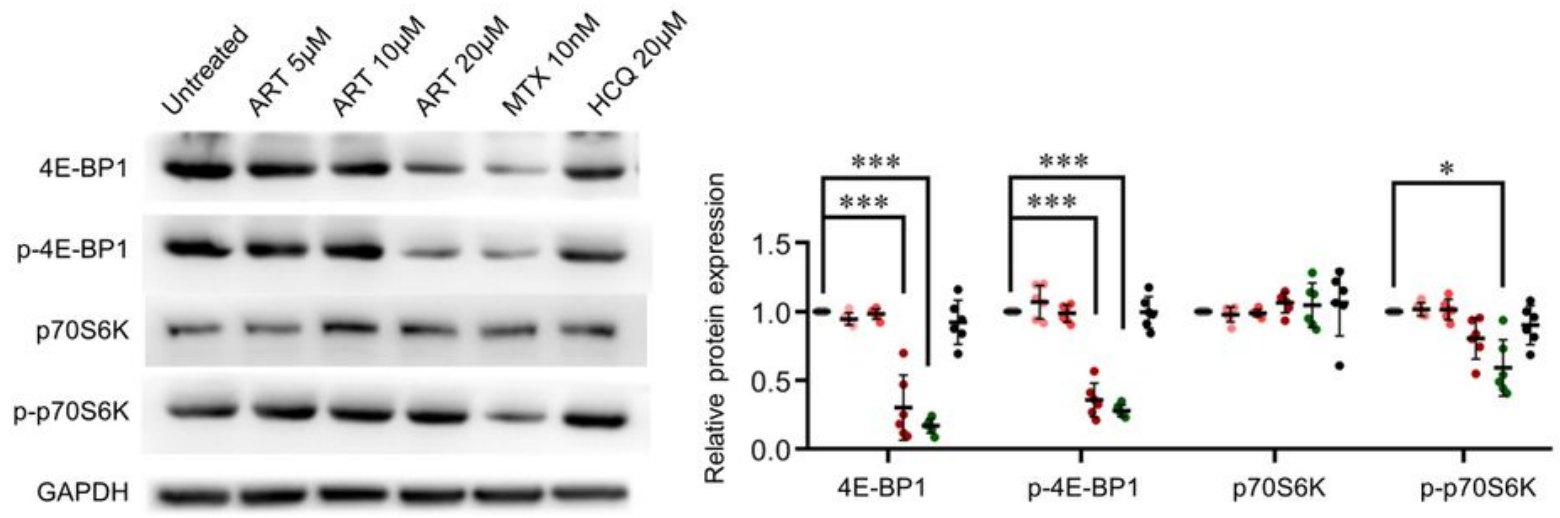

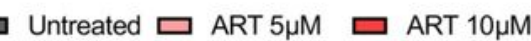

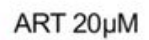

MTX 10nM

$\mathrm{HCQ} 20 \mu \mathrm{M}$ 
Effects of ART on mTOR pathway in RA-FLS. Primary RA-FLS were treated with ART, MTX or HCQ for 24 hours. Expression of mRNA (A) and total protein (B D) of mTOR, p-mTOR (Ser2481), p-mTOR (Ser2448), Raptor, Rictor, G $\beta \mathrm{L}, 4 \mathrm{E}-\mathrm{BP}, \mathrm{p}-4 \mathrm{E}-\mathrm{BP}, \mathrm{p} 70 \mathrm{~S} 6 \mathrm{~K}$ and $\mathrm{p}-\mathrm{p} 70 \mathrm{~S} 6 \mathrm{~K}$ were measured by qPCR and western blot. Relative protein expression was normalized by GAPDH. Data were represented as mean \pm SD from 6 RA patients. ${ }^{* *} \mathrm{P}<0.01,{ }^{* \star *} \mathrm{P}<0.001$, compared with untreated (RA-FLS treated with DMSO). ART: artemether, MTX: methotrexate, HCQ: hydroxychloroquine.

A
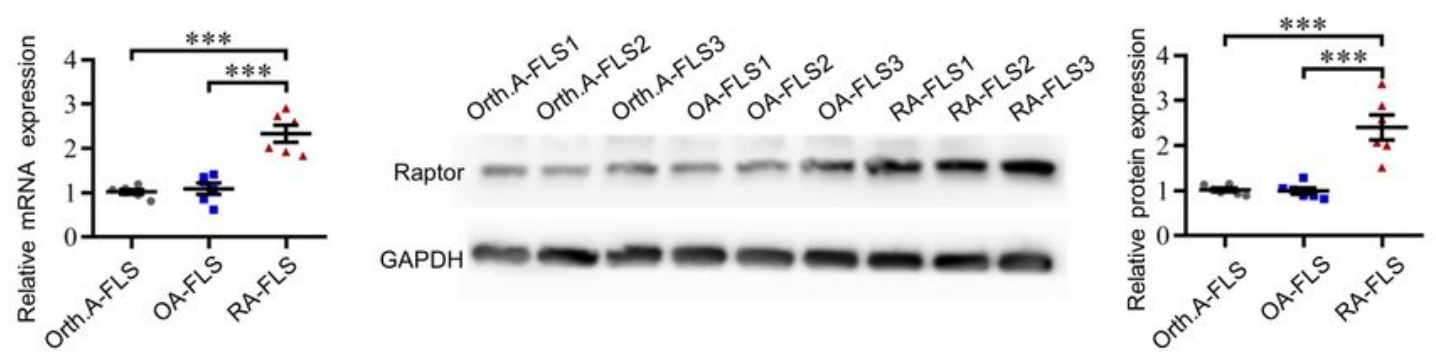

B
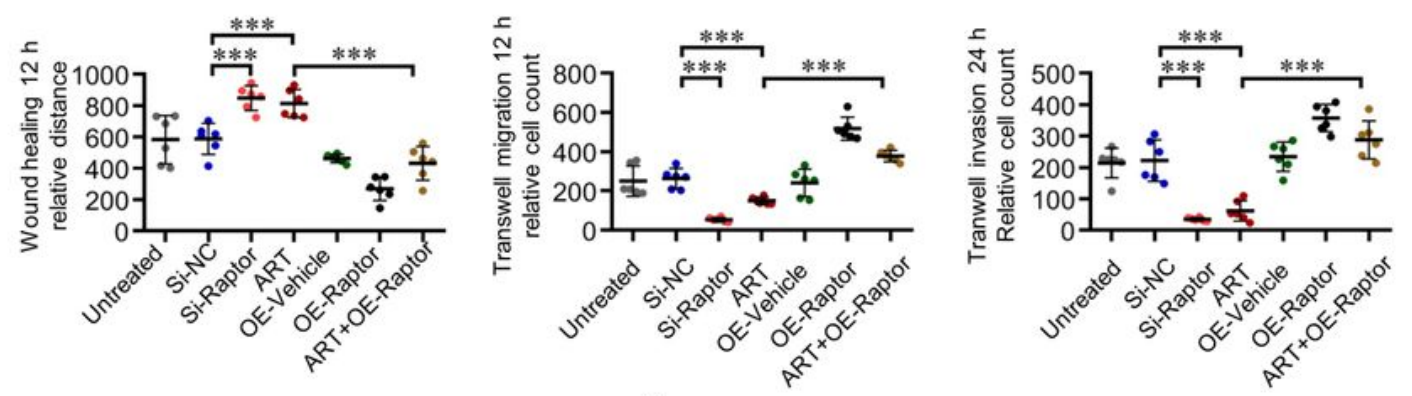

C

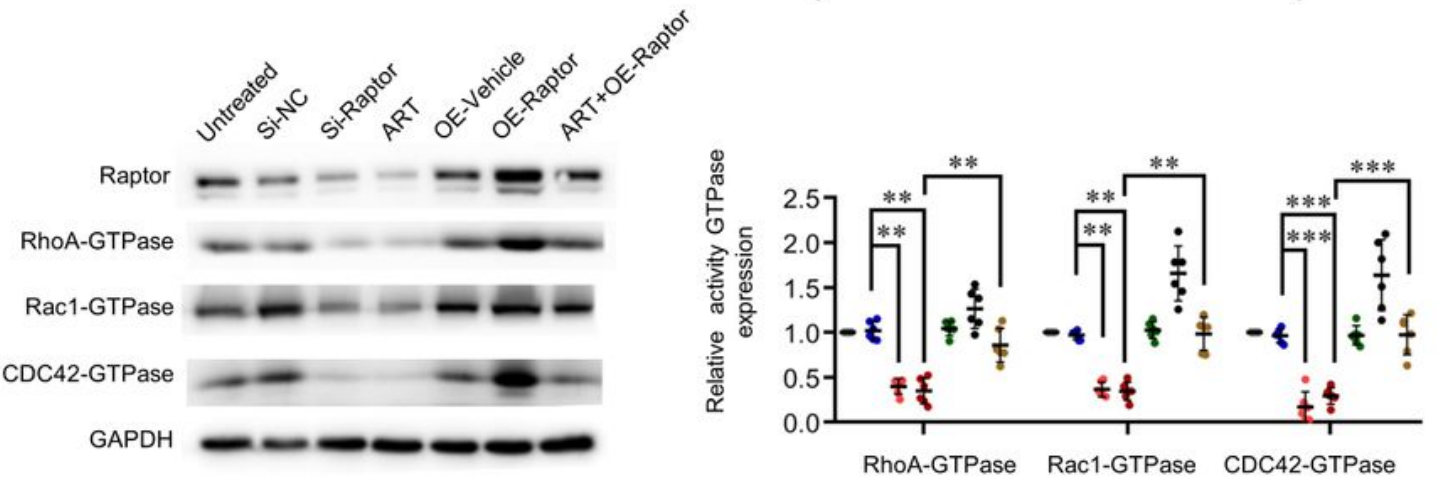

D
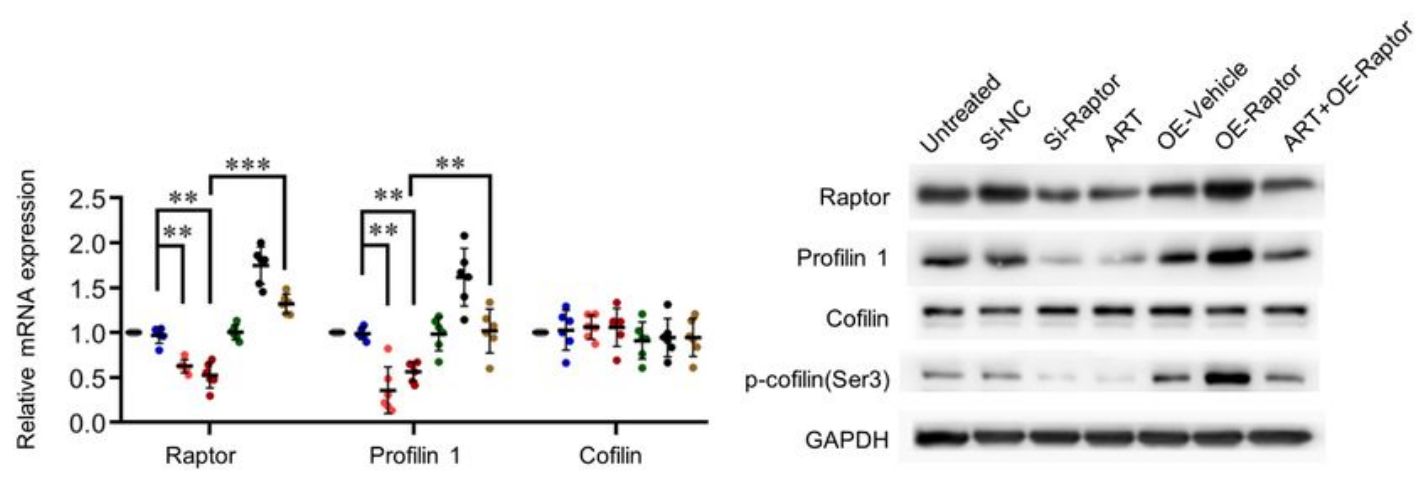

- Untreated

Si-NC $\quad$ Si-Raptor

ART

OE-Vehicle

OE-Raptor - ART+OE-Raptor

Figure 4 
Inhibitory effects of ART on RA-FLS migration and invasion via Raptor pathway. Primary RA-FLS were transfected with siRNA for Raptor knockdown or transfected with lentivirus for Raptor overexpression, and treated with $20 \mu \mathrm{M}$ ART for 24 hours. (A) The qPCR and western blot analysis showed that the expression of Raptor in Orth.A-FLS, OA-FLS and RA-FLS. Data were representative as means \pm SD from 6 Orth.A, 6 OA, and 6 RA patients. $* \star * P<0.001$. (B) Wound healing and transwell assays showed the capacity of horizontal migration, vertical migration and invasion of primary RA-FLS. (C) The activity of RhoA, Rac1 and Cdc42 were measured by pulldown assays. (D) The total protein expression of Raptor, Profilin 1, Cofilin and p-cofilin (Ser3) were measured by western blot. Data were represented as mean \pm SD from 6 RA patients. ${ }^{*} P<0.05,{ }^{*} \mathrm{P}<0.01,{ }^{* \star *} \mathrm{P}<0.001$. NS: non-significant. ART: artemether. 
A

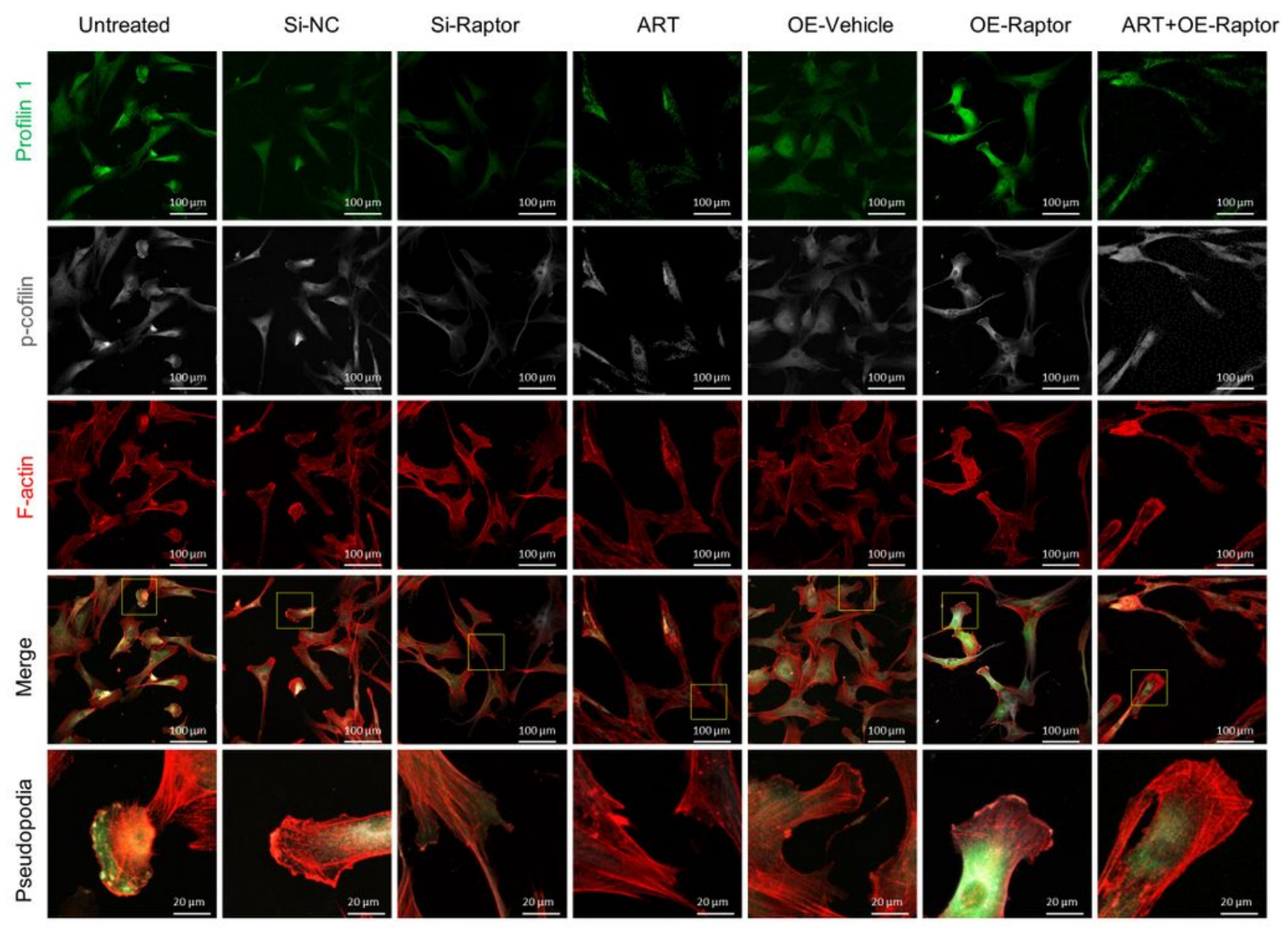

B
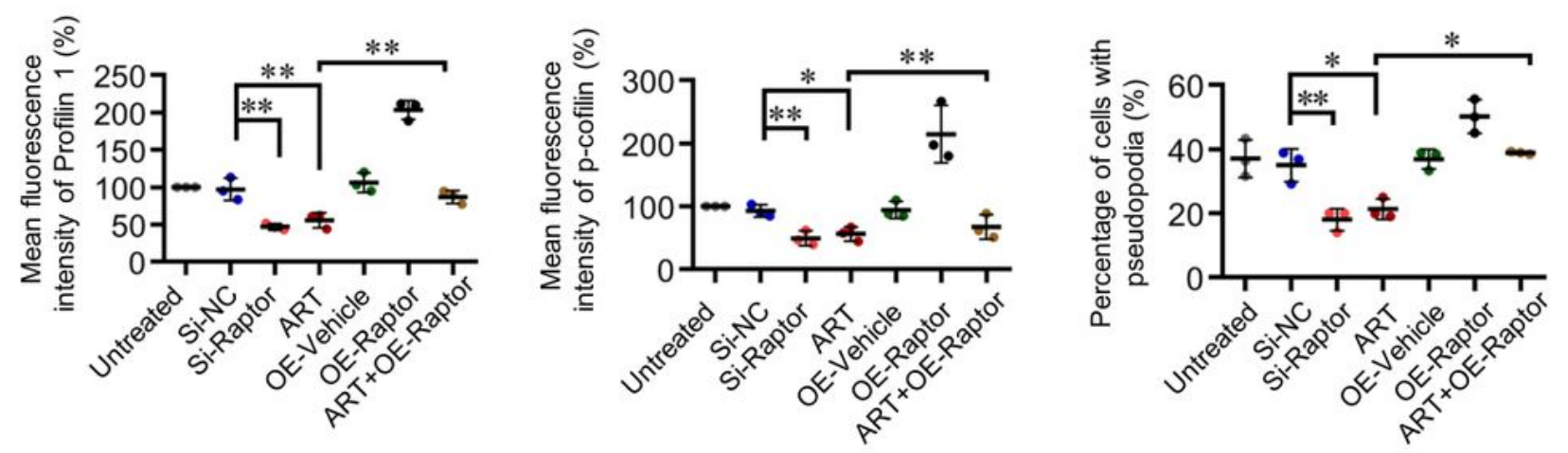

\section{Figure 5}

Inhibitory effects of ART on actin polymerization of RA-FLS via Raptor pathway. Primary RA-FLS were transfected with siRNA for Raptor knockdown or transfected with lentivirus for Raptor overexpression, and treated with $20 \mu \mathrm{M}$ ART for 24 hours. (A) Immunofluorescence staining of Profilin 1, p-cofilin (Ser3) and F-actin were performed in primary RA-FLS. Representative images were shown (original magnification, $\times 200$ above and $\times 1000$ below). The yellow box indicates lamellipodia and filopodia 
formation. (B) Statistics for the mean fluorescence intensity of Profilin 1 or p-cofilin and the percentage of RA-FLS with pseudopodia. Data were represented as means \pm SD from 6 RA patients. ${ }^{*} P<0.05,{ }^{*} P<0.01$. ART: artemether.

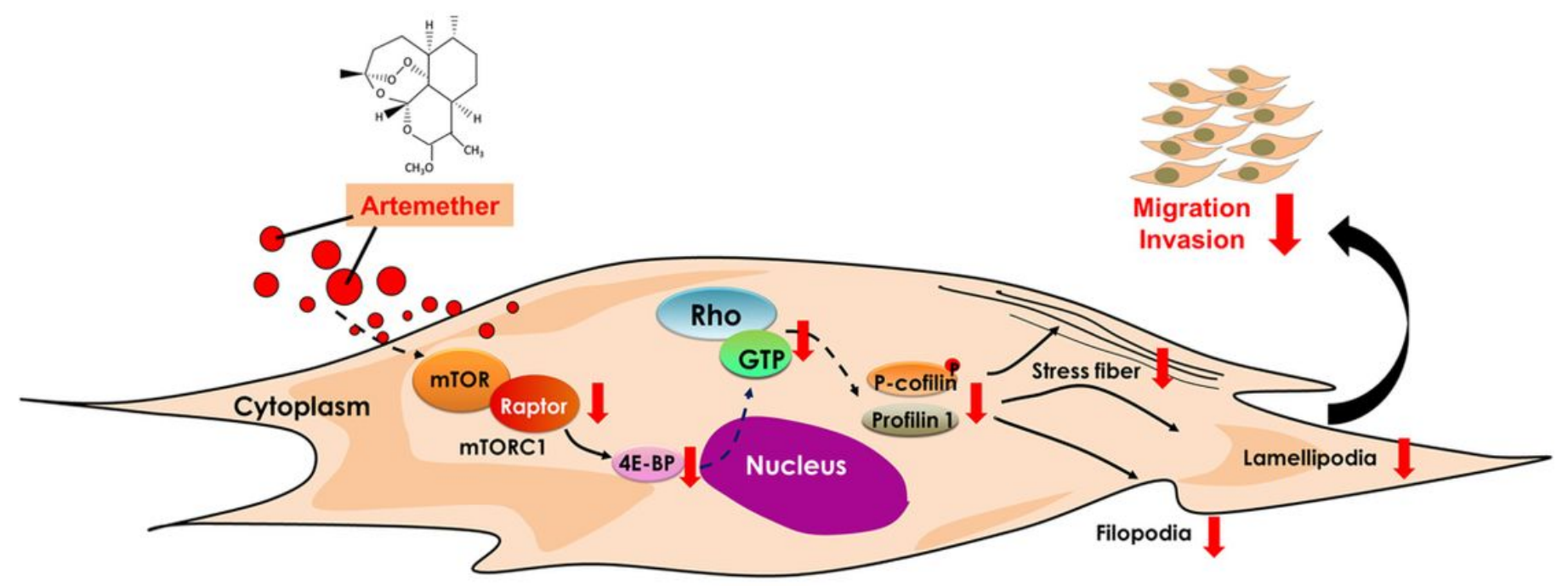

Figure 6

Possible mechanism of ART suppressing migration and invasion of RA-FLS. Artemether (ART) can suppress the expression of Raptor and its downstream target 4E-BP1, as well as the Rho GTPases activity and actin-binding protein expression, then reduce lamellipodia and filopodia formation, leading to the inhibition of migration and invasion of RA-FLS

\section{Supplementary Files}

This is a list of supplementary files associated with this preprint. Click to download.

- Supplementaryinformation.docx 